\title{
Frecuencia de cardiopatías congénitas y adquiridas en 4,544 niños. Estudio basado en ecocardiografía
}

\author{
Frequency of 4,544 children with congenital and acquired heart disease. \\ Echocardiographic study
}

\author{
Jesús De Rubens-Figueroa ${ }^{*}$, Juan C. Ceballos-Cantú ${ }^{2}$ Ana F. López-Ornelas², \\ Mayela Vazquéz-Perdomo ${ }^{3}$ y José L. Pablos-Hach ${ }^{4}$
}

${ }^{1}$ Servicio de Cardiología, Laboratorio de Ecocardiografía, Instituto Nacional de Pediatría; ${ }^{2}$ Curso de pregrado, Facultad de Medicina, Universidad Nacional Autónoma de México; ${ }^{3}$ Residencia de Pediatría, Instituto Nacional de Pediatría; ${ }^{4}$ nstituto Tecnológico y de Estudios Superiores de Monterrey. Ciudad de México, México

\begin{abstract}
Resumen
Objetivo: Mostrar la frecuencia de las cardiopatías congénitas y adquiridas en nuestra institución. Material y métodos: Se realizó un estudio retrospectivo, transversal y descriptivo donde se revisaron las libretas de los estudios ecocardiográficos, realizados en pacientes cuyas edades fueron de recién nacidos a 18 años y se agruparon por tipos de cardiopatías. En el análisis estadístico obtuvimos la frecuencia de ocurrencia, el valor de la proporción de cada cardiopatía. Resultados: En los últimos 10 años ingresaron 4,544 pacientes con cardiopatía. Las 14 cardiopatías más frecuentes correspondieron al 89.21\% de la estadística. La cardiopatía más frecuente fue la persistencia del conducto arterioso con 896 pacientes (19\%) y la cardiopatía adquirida más frecuente fue la miocardiopatía, con 116 pacientes (2.5\%). La tetralogía de Fallot fue la cardiopatía cianógena más frecuente, con 139 pacientes (3\%), a diferencia de otros países. Conclusiones: La frecuencia de cardiopatías congénitas y adquiridas es variable en diferentes áreas étnicas y geográficas.
\end{abstract}

Palabras clave: Frecuencia de cardiopatías. Cardiopatías congénitas. Cardiopatías adquiridas. Cardiopatías cianógenas. Ecocardiografía pediátrica.

\begin{abstract}
Objective: To aim the incidence of congenital and acquired heart disease in our institution. Material and methods: $A$ retrospective, transverse, descriptive and comparative study was conducted, from August 2001 to July 2011, in which we evaluated echocardiograms of patients between the ages of newborn to 18 years of age. They were grouped by heart disease. In the last statistical analysis, we obtained the frequency of occurrence and the proportional value of each cardiopathy. Results: In the last 10 years, we founded 4,544 patients with heart disease. The 14 most common cardiopathies represented $89 \%$ of the statistic. The congenital heart disease most frequent was persistent ductus arteriosus with 896 patients (19\%), and the acquired heart disease most frequent was myocarditis with 116 patients (2.5\%). The tetralogy of Fallot was most frequent
\end{abstract}

\section{Correspondencia:}

*Jesús De Rubens-Figueroa

E-mail: derubens@ @otmail.com CC BY-NC-ND (http://creativecommons.org/licenses/by-nc-nd/4.0/).
Fecha de recepción: 17-04-2020

Fecha de aceptación: 07-11-2020

DOI: 10.24875/ACM.20000166
Disponible en internet: 14-12-2020

Arch Cardiol Mex. (ahead of print) www.archivoscardiologia.com 
congenital heart disease with cyanosis. Conclusions: The frequencies of congenital and acquired heart disease are different in difference geographic and ethnics areas.

Key words: Heart malformation incidence. Congenital and acquired heart disease. Cyanotic heart disease. Pediatric echocardiography. México.

\section{Introducción}

Las cardiopatías congénitas (CC) se presentan en el $5 \%$ de los productos en gestación ${ }^{1}$, muchos de estos fallecen antes del nacimiento. Del 30 al $40 \%$ de los pacientes con muerte fetal presentan alguna alteración cromosómica, las cuales en su gran mayoría cursan con cardiopatía, por lo que las CC son las malformaciones congénitas más frecuentes en el mundo².

En México, en el año 2007 hubo 2,000,198 recién nacidos, de los cuales 17,591 cursaron con CC $(0.8 \%)$, porcentaje de presentación en la población abierta parecido al de otros países ${ }^{3,4}$. La mortalidad en menores de un año a causa de la CC es del $4.7 \% \%^{5}$. Es la primera causa de muerte en países desarrollados y la tercera en países no desarrollados ${ }^{6}$.

La incidencia de CC es muy variable, en el Instituto Nacional de Cardiología (hospital de concentración de enfermedades cardiacas) en una revisión de 120,000 expedientes en 25 años se presentaron 6,000 pacientes con CC $(5 \%)^{6}$. En 1978, en el Hospital General de México, de cada 1,000 pacientes que ingresaron 1.5 fueron con CC $(0.15 \%)^{6}$.

La incidencia de las CC depende de varios factores: el medio ambiente, en donde en algunas zonas geográficas es más frecuente la persistencia del conducto arterioso (PCA); en algunas etnias como en Asia la enfermedad de Kawasaki y en razas caucásicas se presenta el canal atrioventricular (C-AV), la estenosis aórtica (EAo) y la coartación aórtica (CoAo); en cuanto al sexo, en las mujeres es más frecuente la comunicación interauricular (CIA) y la PCA, en los hombres la EAo, la CoAo y la transposición de las grandes arterias (TGA); el bajo peso como en los prematuros la PCA; marcadores genéticos familiares tales como las miocardiopatías ${ }^{2,7,8}$; la edad del paciente al momento del diagnóstico (solo el $50-60 \%$ de las CC se diagnostican en la etapa de recién nacido); si se decide la interrupción del embarazo, si el ecocardiograma es fetal o transtorácico y un adecuado entrenamiento de los pediatras, cardiólogos y radiólogos para realizar un diagnóstico preciso y temprano, con tecnología adecuada para afinar el estudio de la cardiopatía (ecocardiografía modo M, bidimensional-tridimensional, Doppler pulsado, continuo, color, angiocardiografía biplanar, tomografía computarizada, resonancia magnética).

También la incidencia varía al incluir el diagnóstico de pequeñas lesiones cardiacas como son la estenosis pulmonar (EP) mínima, aorta bivalva (Ao biv), ClA pequeña o foramen oval (FOP) y o comunicación interventricular (CIV) pequeña9.

En la Ciudad de México, debido a la altura con respecto al nivel del mar, se han reportado frecuencias de PCA de hasta el $20 \%$ de todos los casos en análisis de 2,257 pacientes con CC en el Hospital de Cardiología del Centro Médico Nacional Siglo XXI; le siguió la CIA (16.8\%); CIV (11\%); tetralogía de Fallot (TF) y atresia pulmonar (AP) con CIV (9.3\%); CoAo y EP (3.6\%, respectivamente), y la conexión anómala total de venas pulmonares (CATVP) $(3 \%)^{10}$.

El objetivo de nuestro trabajo es obtener la frecuencia del tipo de cardiopatías congénitas y adquiridas en pacientes diagnosticados por ecocardiografía (el cual es el método más accesible, con alta sensibilidad y especificidad para el diagnóstico de las cardiopatías), en un instituto pediátrico de concentración de la República Mexicana, en la Ciudad de México.

En México no contamos con un registro exacto de prevalencia y la verdadera incidencia de las CC es difícil de determinar por lo amplio de su definición, sin embargo, de acuerdo con el Registro y Vigilancia Epidemiológica de Malformaciones Congénitas Externas (RYVEMCE) se estima una tasa de 1 por cada 1,000 nacimientos ${ }^{11}$.

\section{Material y métodos}

Se realizó un estudio retrospectivo, longitudinal y descriptivo, donde se revisaron las libretas de registros y los reportes de los estudios ecocardiográficos realizados de septiembre 2001 a octubre 2011. La edad de los pacientes varió de recién nacidos a 18 años de edad.

Como criterios de inclusión se tomaron en cuenta todas las cardiopatías (anormalidad estructural del corazón y de los grandes vasos):

- Adquiridas (cardiopatías que se presentan después del nacimiento). Miocardiopatías (donde incluimos a 
las miocarditis), enfermedades del pericardio, endocarditis, enfermedades de Kawasaki y Takayasu. Esta clasificación se apegó al código de NERICP12.

- Y congénitas (cardiopatías malformadas en la etapa fetal y que se presentan al nacimiento). En estas se analizaron:

- Las acianógenas: PCA (incluso del prematuro), CIV (incluso la muscular), $\mathrm{CIA}$ (incluyendo el FOP), prolapso de la válvula mitral (PVM), Ao biv, C-AV, ventana aortopulmonar, obstrucciones a los tractos de salida derecho e izquierdo, coartación de la aorta y criss cross.

- Dentro de las cardiopatías cianógenas consideramos las siguientes: TF (donde incluimos las AP con CIV), atresia tricuspídea, TGA, síndrome de ventrículo izquierdo hipoplásico (S.VIH), doble salida del ventrículo derecho, ventrículo único (VU) funcional, enfermedad de Ebstein, AP con septo íntegro y malposiciones cardiacas.

Los criterios de exclusión fueron los estudios con diagnósticos dudosos, aquellos que no se discutieron en sesión cardiológica, los ya operados de cirugía correctiva cardiaca, pacientes con arritmias sin malformación cardiaca, con hipertensión arterial sistémica y pulmonar sin cardiopatía y todos aquellos ecocardiogramas que se reportaron con corazón anatómico normal; todos estos se catalogaron en el grupo «sin anomalías anatómicas cardiacas" y no se incluyeron en el estudio.

Muchos de los pacientes con cardiopatía presentan varias lesiones cardiacas asociadas. Para fines de la estadística y comparación con otras instituciones se tomó en cuenta a la lesión anatómica de mayor repercusión hemodinámica.

En los casos de pacientes con varios estudios ecocardiográficos, el diagnóstico final fue hecho con el último ecocardiograma realizado y sometido a revisión en la sesión cardiológica, previo a la corrección de la lesión; en algunos casos se complementó su estudio con angiorresonancia magnética y angiotomografía.

Los estudios de ultrasonografía cardiaca se realizaron con ecocardiógrafos Hewlett Packard 5,500 y en forma posterior con Philips IE33, modo M, bidimensional, Doppler pulsado, continuo, color, con transductores 5-1 y 8-3 MHz.

Los datos recabados se ordenaron en una base de datos y el análisis estadístico consistió en obtener la frecuencia de ocurrencia de cada cardiopatía en estudio, el valor de la proporción obtenida en cada cardiopatía en estudio. Los cálculos se realizaron con Excel 2007 de Microsoft.

\section{Resultados}

En los últimos 10 años se realizaron más de 14,000 ecocardiogramas, de los cuales al restar los repetidos del mismo paciente (seguimientos, procedimientos, controles prequirúrgicos y posquirúrgicos) y los de «sin anomalías anatómicas cardiacas»" resultan 4,544 pacientes con cardiopatía, los cuales se organizaron por tipos de cardiopatía para observar la frecuencia de presentación. Las CC fueron 4,182 (92\%) y las adquiridas fueron $362(8 \%)$. Las CC se manifestaron como acianógenas en 3,684 pacientes (88\%) y cianógenas en 498 (12\%).

El $89.21 \%$ de nuestro estudio está constituido por las 14 cardiopatías más frecuentes; dentro de ellas la PCA fue la más frecuente (19.7\%), con 896 pacientes. El $10.8 \%$ correspondió a otras cardiopatías (Tabla 1).

La cardiopatía cianógena más frecuente fue la TF, con 139 pacientes, que representó el 3.09\% (Fig. 1).

La cardiopatía adquirida más frecuente fueron las miocardiopatías, con 116 casos, que constituyeron el $2.55 \%$ (Fig. 2).

\section{Discusión}

La incidencia de las CC en el recién nacido varía entre el 0.4 y el $1.2 \%, 1,13,14$.

Debido a que el estudio ecocardiográfico se realiza en todos los pacientes con sospecha de cardiopatía y a que hoy en día es el método diagnóstico con mayor sensibilidad, especificidad y de fácil acceso para el paciente (incluso en la misma cama de hospitalización), hemos decidido basar nuestra estadística en los estudios realizados en el Laboratorio de Ecocardiografía y revisado en sesión cardiológica con todo el personal médico.

Más de 6,500 pacientes presentaron hipertensión arterial pulmonar y/o sistémica, arritmias, o corazones anatómicamente normales (sin anomalías cardiacas estructurales); 4,544 presentaron cardiopatía diagnosticada de primera vez y el resto de los ecocardiogramas realizados fueron repeticiones de control.

\section{Frecuencia de cardiopatías (Tabla 1)}

En nuestro estudio incluimos a los niños prematuros y recién nacidos de término con PCA, la mayoría con insuficiencia cardiaca e incluso con decisión de manejo farmacológico, el cual fue con indometacina.

También se incluyeron las CIV musculares, ya que es un diagnóstico que requiere vigilancia cardiológica. 
Tabla 1. Frecuencia de cardiopatías congénitas y adquiridas

\begin{tabular}{|l|l|c|}
\hline N. & Cardiopatía & Frecuencia (\%) \\
\hline 1 & Persistencia de conducto arterioso & $896(19.72)$ \\
\hline 2 & Comunicación interventricular & $788(17.34)$ \\
\hline 3 & Comunicación interauricular & $668(14.70)$ \\
\hline 4 & Estenosis pulmonar & $263(5.79)$ \\
\hline 5 & Estenosis aórtica & $235(5.17)$ \\
\hline 6 & Coartación aórtica & $210(4.62)$ \\
\hline 7 & Foramen oval permeable & $200(4.40)$ \\
\hline 8 & Tetralogía de Fallot & $139(3.06)$ \\
\hline 9 & Miocardiopatía & $116(2.55)$ \\
\hline 10 & Pericarditis & $114(2.51)$ \\
\hline 11 & Conexión anómala total de venas pulmonares & $110(2.42)$ \\
\hline 12 & Malformaciones de la válvula tricúspide & $108(2.38)$ \\
\hline 13 & Enfermedad de Kawasaki & $104(2.29)$ \\
\hline 14 & Malformaciones de la válvula mitral & $103(2.27)$ \\
\hline $04 a 8$ & & \\
\hline
\end{tabular}

\section{Otras:}

\begin{tabular}{|c|c|c|}
\hline 15 & Canal auriculoventricular & $92(2.02)$ \\
\hline 16 & Doble salida de ventrículo derecho & $73(1.61)$ \\
\hline 17 & Transposición de grandes arterias & $60(1.32)$ \\
\hline 18 & Atresia tricuspídea & $44(0.97)$ \\
\hline 19 & Aorta bivalva & $43(0.95)$ \\
\hline 20 & Prolapso de válvula mitral & $28(0.62)$ \\
\hline 21 & Atresia pulmonar & $28(0.62)$ \\
\hline 22 & Endocarditis & $18(0.40)$ \\
\hline 23 & Tumores cardiacos & $14(0.31)$ \\
\hline 24 & Malposiciones cardiacas & $12(0.26)$ \\
\hline 25 & Síndrome de ventrículo izquierdo hipoplásico & $12(0.26)$ \\
\hline 26 & Doble salida de ventrículo izquierdo & $10(0.22)$ \\
\hline 27 & Insuficiencia aórtica & $10(0.22)$ \\
\hline 28 & Enfermedad de Takayasu & $10(0.22)$ \\
\hline 29 & Insuficiencia pulmonar & $9(0.20)$ \\
\hline 30 & Criss cross & $6(0.13)$ \\
\hline 31 & Anomalía de arterias sistémicas & $6(0.13)$ \\
\hline 32 & Ventrículo único verdadero & $5(0.11)$ \\
\hline 33 & Dilatación de la raíz aórtica & $5(0.11)$ \\
\hline 34 & Tronco arterioso común & $4(0.09)$ \\
\hline \multirow[t]{2}{*}{35} & Ventrículo derecho hipoplásico & $1(0.02)$ \\
\hline & Total & $4,544(100)$ \\
\hline
\end{tabular}




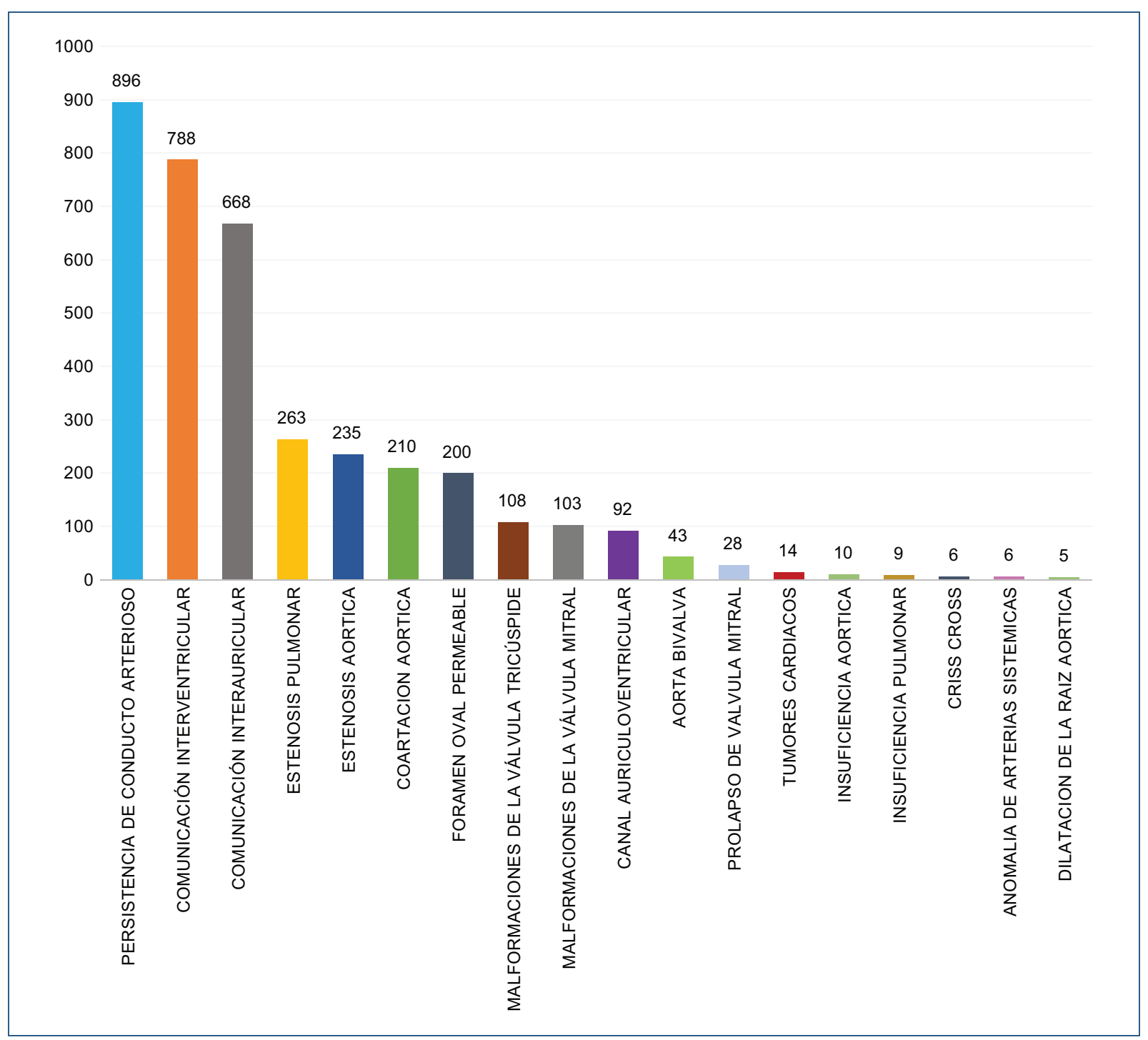

Figura 1. Frecuencia de las cardiopatías acianógenas.

Muchos de los pacientes con CIA cursaron asintomáticos, sin embargo, es de utilidad realizar el diagnóstico ecocardiográfico, ya que la mayoría se trata en la etapa pediátrica. Aquí se incluyeron cinco pacientes con conexión anómala parcial de las venas pulmonares (dos correspondieron a síndromes de "cimitarra»).

En los rubros de anormalidades de la válvula pulmonar incluimos: estenosis, insuficiencias de moderadas a severas, dos pacientes con agenesia de valvas pulmonares, una pulmonar bivalva y 18 pacientes con estenosis de ramas pulmonares.

En las anormalidades de la válvula aórtica se incluyeron estenosis valvulares y subvalvulares, insuficiencias de moderadas a severas e incluso estenosis supravalvular aórtica. En los últimos años hemos notado el incremento de disfunciones valvulares, las cuales muchas de ellas correspondieron a endocarditis de origen inmunológico.

El diagnóstico ecocardiográfico de FOP en la edad pediátrica es de utilidad en el seguimiento médico hasta la etapa adulta, por las complicaciones que pueden presentarse (enfermedad vascular cerebral o migrañas), así como las CIV pequeñas pueden no cerrar espontáneamente entre el 30 y el $50 \%$ de los $\operatorname{casos}^{9}$, y requerir de profilaxia antibiótica para endocarditis infecciosa.

Las anormalidades de la válvula tricúspide incluyeron estenosis, insuficiencias de moderadas a severas e incluso se incluyeron 33 pacientes con enfermedad de Ebstein. 


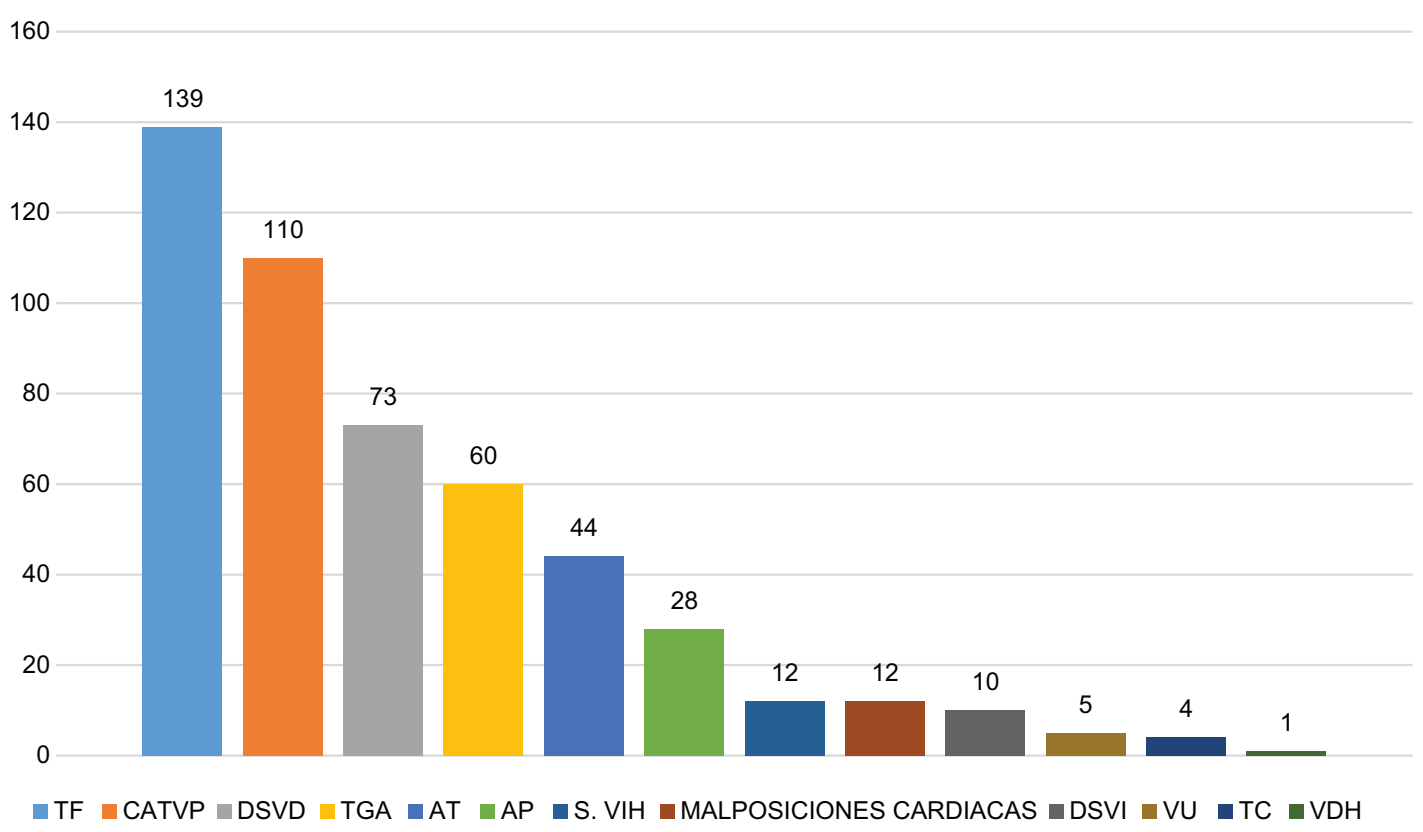

Figura 2. Frecuencia de las cardiopatías cianógenas.

TF: tetralogía de Fallot; CATVP: conexión anómala total de venas pulmonares; DSVD: doble salida de ventrículo derecho; TGA: transposición de grandes arterias; AT: atresia pulmonar; S.VIH: síndrome de ventrículo izquierdo hipoplásico; VU: ventrículos únicos funcionales; TC: tronco común; VDH: ventrículo derecho hipoplásico.

En anormalidades de la válvula mitral se incluyeron: estenosis, insuficiencias moderadas a severas y dobles lesiones, incluyendo dos casos de afección reumática aguda.

Dentro del grupo denominado "otros", la CIA tipo ostium primum se incluyó en los C-AV. No es muy frecuente, debido a que la cardiopatía más frecuente en nuestro medio en los niños con síndrome de Down no es el C-AV ${ }^{15}$.

Los pacientes con Ao biv se diagnosticaron por imagen ecocardiográfica; con sospecha de cardiopatía, la mayoría cursaron asintomáticos, por lo que algunos pacientes con este diagnóstico no son diagnosticados hasta una vez iniciado los síntomas en la etapa adulta. Estos niños tienen la necesidad de seguimiento clínico, para evitar las complicaciones que puedan presentarse y llevar a cabo profilaxia antibiótica contra endocarditis infecciosa.

Los pacientes con AP fueron incluidos solo con septum íntegro.

De los tumores, 13/14 fueron rabdomiomas (la tumoración cardiaca más frecuente en pediatría), asociados a pacientes con esclerosis tuberosa.

Las malposiciones correspondieron a las dextrocardias e isomerismos, algunos con ventrículos únicos.
En nuestra estadística obtuvimos solo nueve pacientes con S.VIH, tres de estos con atresia mitral; cantidad poco frecuente en nuestro medio a diferencia de lo publicado en otros países ${ }^{4,12,16}$. Los cinco pacientes con VU correspondieron a dobles entradas ventriculares. Las anormalidades de arterias sistémicas correspondieron dos a alteraciones vasculares de los vasos supraaórticos (anillos vasculares) y cuatro con interrupción del arco aórtico.

En nuestro estudio encontramos que en los últimos 10 años la cardiopatía más frecuente fue la PCA, a diferencia de otras casuísticas como la de Boston, donde casi todas coinciden en que la CIV es la cardiopatía más frecuente ${ }^{12}$. Otras excepciones fueron estudios como el de Sudáfrica ${ }^{17}$, que coincide con nuestro estudio, respecto a que la PCA es la más frecuente y el de Sri Lanka, donde se publicó que la cardiopatía más frecuente fue la $\mathrm{CI} \mathrm{A}^{18,19}$; las siguientes cardiopatías publicadas con mayor frecuencia de presentación en la mayoría de los estudios fueron la CIA o PCA, EP, TF, EAo, CoAo y TGA ${ }^{20,21}$; solo en aborígenes de Australia ${ }^{22}$ como tercera cardiopatía más frecuente se encontró la CATVP, cardiopatía que es muy frecuente en nuestro instituto en México, donde ocupa el 2 . $^{\circ}$ lugar de las cianógenas (Tabla 1). 


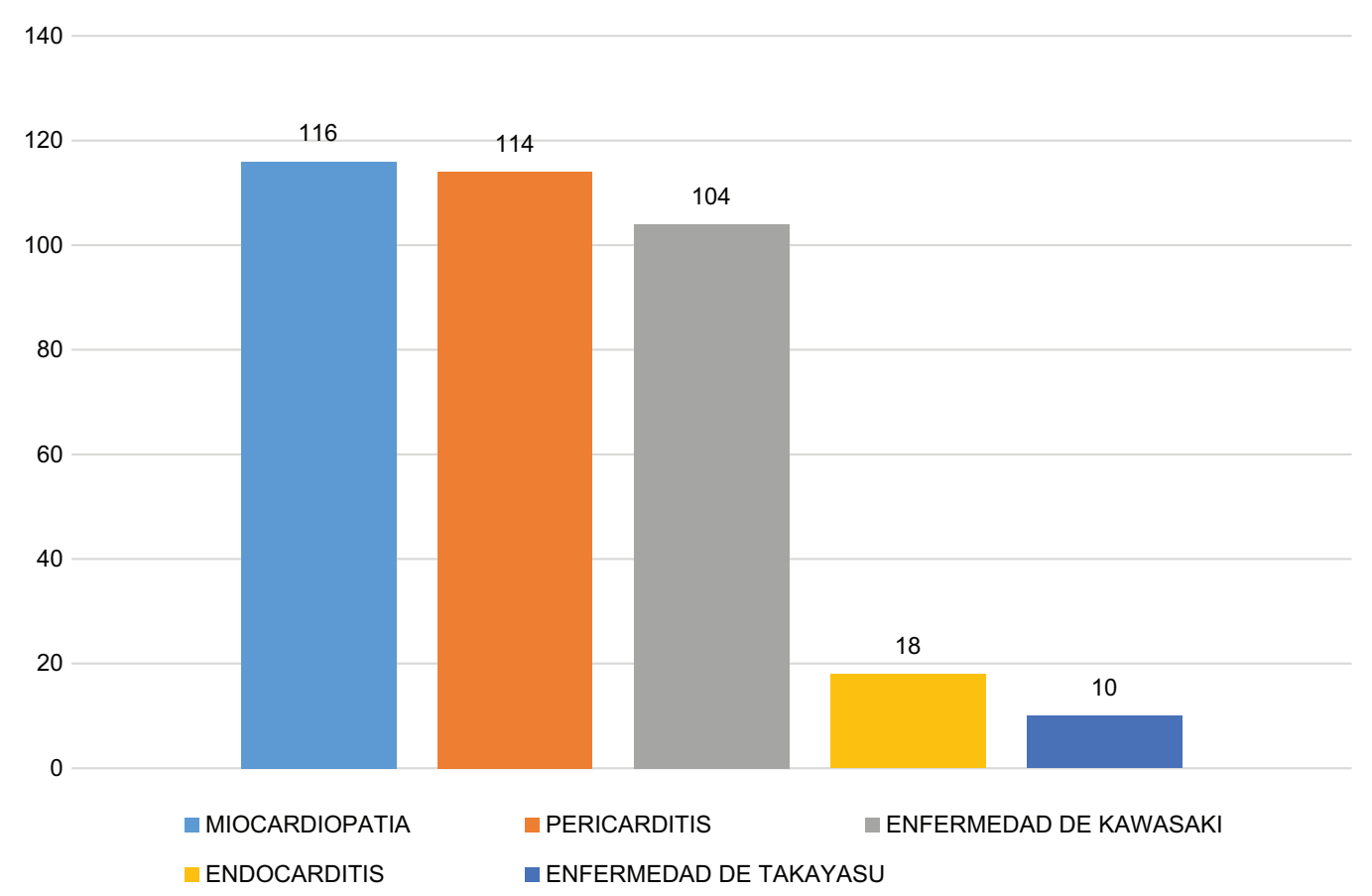

Figura 3. Frecuencia de las cardiopatías adquiridas.

Es de hacerse notar que algunos estudios publicados no incluyen al PVM ni a la Ao biv, por ser estudios previos a la era de la ecocardiografía, que con el advenimiento de este estudio ha incrementado el diagnóstico de estas cardiopatías, sí incluidas en este estudio y en las últimas publicaciones de la literatura mundial $\left.\right|^{2,7,20,21,23}$. Con los avances tecnológicos y de los conocimientos médicos, cada día hay más niños prematuros que sobreviven y presentan frecuentemente manifestaciones de PCA, incrementándose la estadística de esta cardiopatía, aunado a la altitud de 2,250 msnm de la Ciudad de México (Fig. 1).

\section{Cardiopatías cianógenas (Fig. 2)}

La TF es la CC cianógena más frecuente en nuestro medio (se incluyeron las AP con CIV), le sigue la CATVP por encima de otras publicadas en otros países, como son la TGA y el S.VIH. Llama la atención la presentación de 10 casos de doble salida del ventrículo izquierdo (cardiopatía muy rara en la literatura mundial) $)^{12,19,24}$.

\section{Cardiopatías adquiridas (FIG. 3)}

Las más frecuentes fueron las anormalidades pericárdicas (pericarditis, derrames y o tamponades) (muchas de ellas secundarias a enfermedades inmunológicas) y las anomalías miocárdicas.

En las enfermedades miocárdicas se incluyeron los cuatro tipos de miocardiopatías, incluso dos pacientes con miocardiopatía compactada, tres casos de miocardiopatía restrictiva y el resto fueron hipertróficas y dilatadas, muchas de estas secundarias a enfermedades metabólicas, musculares, neurológicas, infecciosas y a efectos secundarios a quimioterápicos, se incluyeron 22 casos de miocarditis. Quizá la frecuencia está influenciada por ser nuestro instituto un hospital de especialidades pediátricas.

La frecuencia de presentación de la enfermedad de Kawasaki ha incrementado en los últimos años (para llegar a ser pronto la cardiopatía adquirida de más frecuencia); de nuestros 95 pacientes, 45 presentaron alteración de coronarias.

La mayoría de los casos de endocarditis fueron de etiología inmunológica y en menor frecuencia de origen infeccioso. No encontramos ningún caso de fiebre reumática.

La arteritis de Takayasu es una enfermedad por definición de la etapa adulta, sin embargo, en nuestra frecuencia diagnosticamos en la edad pediátrica aproximadamente un paciente por año.

Consideramos útiles este tipo de estadísticas y que en cuanto mejoren los avances tecnológicos y la experiencia 
ecocardiográfica para el diagnóstico de las CC habrá una mejor prevención y tratamiento de estas.

\section{Conclusiones}

En nuestro instituto:

- La CC más frecuente es la PCA, seguida de la CIV, esta última es la cardiopatía más frecuente en otros países.

- La cardiopatía cianógena más frecuente es la TF, seguida de la CATVP, esta última a diferencia de otras publicaciones en donde se menciona la TGA.

- Las cardiopatías adquiridas más frecuentes son las enfermedades pericárdicas y las del miocardio, notando un incremento en la frecuencia de presentación de la enfermedad de Kawasaki.

- La frecuencia de cardiopatías congénitas y adquiridas varía de acuerdo con las zonas geográficas y diversas etnias.

El estudio tiene limitantes: es retrospectivo, fue llevado a cabo por tres observadores, se tomaron los datos de los registros de libreta de ecocardiográfica, con diagnósticos finales que se revisaron finalmente en las sesiones que se obtuvieron en conjunto con el personal de cardiología.

El ecocardiograma es muy útil como estudio diagnóstico de las cardiopatías congénitas y adquiridas y la mayoría de las veces proporciona el diagnóstico final y sus características, aunque en ocasiones tiene algunas limitantes.

\section{Financiación}

La presente investigación no ha recibido ninguna beca específica de agencias de los sectores público, comercial o sin ánimo de lucro.

\section{Conflictos de intereses}

Los autores declaran no tener conflicto de intereses.

\section{Responsabilidades éticas}

Protección de personas y animales. Los autores declaran que para esta investigación no se han realizado experimentos en seres humanos ni en animales.

Confidencialidad de los datos. Los autores declaran que han seguido los protocolos de su centro de trabajo sobre la publicación de datos de pacientes.
Derecho a la privacidad y consentimiento informado. Los autores declaran que en este artículo no aparecen datos de pacientes.

\section{Bibliografía}

1. Hoffman IEJ, Kaplan S. The incidence of congenital heart disease. J Am Coll Cardiol. 2002;39:1890-900.

2. Martínez OP, Romero IC, Alzina AV. Incidencias de las cardiopatías congénitas en Navarra. Rev Esp Cardiol. 2005;58:1428-34.

3. Mitchell SC, Korones SB, Berendes HW. Congenital heart disease in 56,109 births. Incidence and natural history. Circulation. 1971:43:323-32.

4. Samánek M, Slavíd Z, Zborilová B, Hrobonová V, Vorísková M, Skovránek J. Prevalence, treatment, and outcome of heart disease in live-born children: A prospective analysis of 91,823 live-born children. Pediatr Cardiol. 1989;10:205-11.

5. Ramírez MS. Retos en el cuidado de los niños con cardiopatías congénitas en México (INEGI). Memorias del $2 .^{\circ}$ Congreso Nacional de la Asociación Mexicana de Especialistas en Cardiopatías Congénitas, Zacatecas, Zac., México. 2010; Ag. 12-14.

6. Espino Vela VJ. Cardiología pediátrica. 3. ${ }^{a}$ ed. México: Editorial Méndez; 1994. pp. 41-8

7. Marelli JA, Mackie SA, Ionescu-Ittu R, Rahme E, Pilote L. Congenital heart disease in the general population. Circulation. 2007:115:163-72.

8. Mendieta G, Santiago E, Mendieta H, Dorantes R, Ortiz G, Otero G. Incidencia de las cardiopatías congénitas y los factores asociados a la letalidad en niños nacidos en dos hospitales del Estado de México. Gac Med Mex. 2013;149:617-23.

9. Hoffman IEJ, Kaokab S, Liberthson RR. Prevalence of congenital heart disease. Am Heart J. 2004;147:425-39.

10. Vega T, Gonzalez G, Llanes MC, Ley L, Gari M, García Y. Morbilidad y mortalidad por cardiopatías congénitas en lactantes. Villa Clara, años 2006-2010. Acta Médica del Centro. 2011;5(4).

11. Mexican Registry and Epidemiological Surveillance of External Congenital Malformations. Mexico: RYVEMCE. En: International Clearing House of Birth Defects Surveillance and Research. Annual Report 2010 with data for 2008 [Internet]. International Clearing House of Birth Defects Surveillance and Research; 2010. Disponible en: http://www.icbdsr.org/ wp-content/annual_report/Report2010.pdf

12. Report of the New England Regional Infant Cardiac program. Pediatrics. 1980;65(Suppl):377-461.

13. Ferencz C, Rubin JD, McCarter RJ, Boughman JA, Wilson PD, Brenner Jl, et al. Cardiac and non-cardiac malformations: observations in a population-based study. Teratology. 1987:35:367-78.

14. Manetti A, Pollini I, Cecchi F, et al. Epidemiologia delle malformazioni cardiovascolari. III. Prevalenza e decorso in 46,895 nati vivi all maternita di Careggi, Firenze, nel periodo 1975-1984. G Ital Cardiol. 1993;23: 145-52.

15. De Rubens FJ, Del Pozzo MB, Pablos H JL, Calderón JC, Castrejón UR. Malformaciones cardiacas en los niños con síndrome de Down. Rev Esp Cardiol. 2003;56:894-9.

16. Hoffman IEJ. Incidence of congenital heart disease: I. Postnatal incidence. Pediatr Cardiol. 1995;16:103-13.

17. Van der Horst RL, Winship WS, Pitaway D, Gibb BH, Lapinsky GB. Congenital heart disease in the South African Bantu: a report of 117 cases. S Afr Med J. 1968;42:1271-3.

18. Wallooppillai NJ, Jayasinghe MD. Congenital heart disease in Ceylon. $\mathrm{Br}$ Heart J. 1970;32:304-6.

19. Pérez-Lescure PJ, Mosquera GM, Latasa ZP, Crespo Marcos D. Incidencia y evolución de las cardiopatías congénitas en España durante 10 años (2003-2012). An Pediatr (Barc). 2018;89(5):294-301.

20. Baltaxe $E$, Zarante I. Prevalencia de malformaciones cardiacas congénitas en 44,985 nacimientos en Colombia. Arch Cardiol Mex. 2006;76:263-8.

21. Triedman KJ. Methodologic issues for database development: trends. En: Keane FJ, Lock EJ, Fyler CD. NADA'S Pediatric Cardiology 2th Ed. Philadelphia, Penn: Sanders-Elsevier; 2006. pp. 323-336.

22. Tofler OB. Congenital heart disease in aboriginals. Med $\mathrm{J}$ Aust. 1979;1:620.

23. Nicholls M. UK congenital heart centres under scrutiny. Circulation. 2007; 116:73-5.

24. Abid D, Elloumi A, Abid L, Mallek S, Aloulou H, Chabchoub I, et al. Congenital heart disease in 37,294 births in Tunisia: Birth prevalence and mortality rate. Cardiol Young. 2014;24:866-71. 